Mutanwad et al., 2019

\title{
O-glycosylation of SPL transcription factors regulates plant developmental transitions downstream of miR156
}

Krishna Vasant Mutanwad, Nicole Neumayer, Claudia Freitag ${ }^{\mathrm{a}}$, Isabella Zangl, and Doris Lucyshyn*

Department of Applied Genetics and Cell Biology, University of Natural Resources and Life Sciences, Vienna, Muthgasse 18, 1190 Vienna, Austria.

${ }^{a}$ Current address: Department of Cardiology, Medical University Vienna, Währinger Gürtel 18-

20, 1090 Vienna

"Correspondence: doris.lucyshyn@boku.ac.at

Keywords: Arabidopsis thaliana, O-glycosylation, plant development, phase change, flowering time, SPL

\section{SUMMARY}

The timing of plant developmental transitions is decisive for reproductive success and thus tightly regulated by a number of pathways with a high degree of crosstalk between them. Such complex regulatory pathways often involve post-translational modifications (PTMs), integrating internal and environmental signals. O-glycosylation, the attachment of a single monosaccharide to serine or threonine of nuclear and cytosolic proteins, is one of these PTMs, affecting a number of very diverse proteins. Here we show that mutants in the $\mathrm{O}$ fucosyltransferase SPINDLY (SPY) show accelerated developmental transitions. In plants, the transition from juvenile to adult and later to reproductive phase is controlled by an endogenous pathway regulated by miR156, targeting the SQUAMOSA PROMOTER BINDING PROTEIN (SBP/SPL) family of transcription factors. SPLs regulate a number of developmental processes, such as trichome formation, leaf shape, leaf growth rate and floral transition. We present genetic analysis showing that O-glycosylation regulates transitions independently of miR156 levels, but depending on functional SPLs. Moreover, SPLs interact directly with SPY and are O-glycosylated. Our results suggest a model where O-glycosylation is involved at several steps in the regulation of developmental transitions, and plays an important role in fine-tuning different regulatory pathways.

\section{INTRODUCTION}

During their life cycle, plants undergo developmental transitions by changing from the juvenile to adult and later the reproductive phase (Poethig 2003; Baurle and Dean 2006; Huijser and Schmid 2011). Each of these irreversible transitions is marked by specific morphological and developmental changes, and while they are governed by an internal developmental program, the timing is flexible and regulated by the environment. Both of these transitions are controlled by a group of small RNAs, the families of miR156 and miR172. miR156 directly targets the SQUAMOSA PROMOTER BINDING PROTEIN (SBP/SPL) family of transcription factors, which then control both transitions by inducing a number of transcription factors regulating adult leaf traits, floral transition and floral meristem identity, as well as expression of miR172 (Schwarz et al. 2008; Poethig 2009; Wang et al. 2009; Wu et al. 2009; Yang et al. 2011; Jung et al. 2012; Yu et al. 2012; Fouracre and Poethig 2016; Hyun et al. 2016; Xu et al. 2016). Interestingly, regulation of SPLs by miR156 occurs both by transcript cleavage as well as translation repression (He et al. 2018), reflecting the high level of complexity in the regulatory pathways of developmental transitions. Additionally, the decrease in miR156 expression over 
the course of development is strongly influenced by metabolism: the accumulation of sugars during plant growth leads to a decrease in miR156 and thus an increase in miR172, which then additionally feeds back negatively on the expression of miR156 (Yang et al. 2013; Yu et al. 2013). Thereby as plants grow and build up biomass, the increasing availability of sugars leads to a release of the repression of SPL transcription factors and thus the developmental switch from the juvenile to adult and later reproductive phase.

The decision of when to flower is extremely important for plants to ensure reproductive success, and thus the transition from the vegetative to the reproductive phase is controlled not only by the internal developmental program described above, but also by seasonal cues and environmental conditions, such as day length, ambient temperature or light quality, which are integrated at several levels. Environmental factors, most importantly day length, are perceived in the leaves, where they induce the expression of the floral integrator $F T$ (FLOWERING LOCUS T). FT is a small, mobile protein, moving through the vasculature to the shoot apical meristem, where it interacts with the transcription factor Flowering Locus $D$ (FD) to induce flowering (Wigge et al. 2005; Jaeger and Wigge 2007; Mathieu et al. 2007). In the shoot apical meristem, the floral integrators SOC1 (SUPRESSOR OF OVEREXPRESSION OF CONSTANTS 1), LFY (LEAFY) and FT regulate the transcriptional network that underlies flowering time control, as reviewed in (Andres and Coupland 2012). A role for gibberellin in the induction of flowering time has already been suggested in the 1950s, and work with the long-day plant Arabidopsis thaliana has later shown that gibberellin is necessary for floral induction under short photoperiods (Wilson et al. 1992), while in long days its effect is mostly masked by the much earlier responding photoperiod pathway (Reeves and Coupland 2001). Gibberellins have since been placed at several points within the flowering time regulatory network (Moon et al. 2003; Achard et al. 2004; Jung et al. 2012; Porri et al. 2012; Yu et al. 2012; Yamaguchi et al. 2014; Galvao et al. 2015; Hyun et al. 2016), with SOC1, $L F Y$ and/or SPLs being direct targets. The effect of gibberellins on flowering time is mediated by DELLA proteins, a small family of transcriptional repressors that are negatively regulated by gibberellin (Harberd et al. 2009). In the absence of gibberellin, DELLA proteins bind to a number of transcription factors, thereby preventing their ability to bind their target genes (de Lucas et al. 2008; Feng et al. 2008; Li et al. 2012). The miR156-regulated aging pathway is also affected by gibberellins, as SPL15 is directly inhibited by the interaction with the DELLA protein RGA in the absence of gibberellin (Hyun et al. 2016).

O-glycosylation of nucleocytoplasmic proteins is an abundant post-translational modification, with a number of very diverse targets. In plants, the O-GIcNAc Transferase (OGT) SECRET AGENT (SEC), and the Protein O-Fucosyltransferase (POFUT) SPINDLY (SPY) are described. These enzymes use UDP-GIcNAc or GDP-fucose respectively to transfer the respective single sugar moiety to serine or threonine residues on their target proteins, among them are the gibberellin signaling repressing DELLA proteins as well as a number of other transcriptional regulators (Zentella et al. 2016; Xu et al. 2017; Zentella et al. 2017). While sec-mutants show only very subtle phenotypes, spy-mutants show a range of developmental defects, and most of them have been explained by enhanced gibberellin signaling (Swain et al. 2001; Tseng et al. 2001; Silverstone et al. 2007; Zentella et al. 2016; Zentella et al. 2017). One of the most prominent features of spy-mutants is early flowering. In long photoperiods, SPY acts together with GIGANTEA $(\mathrm{GI})$ to repress expression of CONSTANS (CO) and FT (Tseng et al. 2004). SPY also strongly represses flowering in short photoperiods, and so far this has been explained by its role in regulating gibberellin (GA) signaling (Swain et al. 2001; Silverstone et al. 2007). 
Here, we present a further characterization of the role of SPY in the control of floral transitions, and show an additional function of O-glycosylation in the regulation of the transition from juvenile to adult to reproductive phase. A combination of genetic and phenotypic analysis suggests a direct regulation of SPL-transcription factors downstream of miR156 by Oglycosylation, potentially independent of gibberellins, with redundancy between O-GIcNAc and $\mathrm{O}$-fucose modification, but a much stronger effect of $\mathrm{O}$-fucosylation.

\section{RESULTS}

\section{The O-fucosyltransferase SPINDLY delays flowering in long and short photoperiods}

In order to characterize the effect of O-glycosylation on flowering time regulation, we did a detailed genetic and phenotypic analysis. As strong spy alleles show severely reduced fertility (Silverstone et al. 2007; Zentella et al. 2017), we used the T-DNA-insertion line spy-22 (SALK_090582) for all our experiments. This line showed strongly reduced expression of SPY and was flowering early at $8.6 \pm 1.4$ total rosette leaves (TRL), compared to $13.1 \pm 1.1$ in the wild-type Col-0 (Figure 1A-B, Fig. S1A-B, Table 1). The early flowering of spy-22 was complemented by a SPY::SPY:Flag (SPY:Flag) construct (Fig.S1C-D). Recently, slight early flowering of sec-5 was reported (Xing et al. 2018), which we also observed, however with a very weak difference to the wild-type $(11.8 \pm 1.6 \mathrm{TRL}$ in sec-5 compared to $13.1 \pm 1.1$ in Col0 , see Figure $1 \mathrm{~A}$ and Table 1). Accordingly, transcript levels of the major floral integrator gene $F T$ were up-regulated in spy-22 but not sec-5, (Figure $1 \mathrm{~B}$ ). When grown in short photoperiods (8 h light / $16 \mathrm{~h}$ dark), where $F T$ is not expressed, spy-22 also displayed strong early flowering with 22.7 $\pm 0.5 \mathrm{TRL}$ compared to $66 \pm 7.9$ in Col-0, which we did not observe in sec-5, flowering at $61.0 \pm 5.0 \mathrm{TRL}$ (Figure 1C, Table 1). Similarly, early flowering of $s p y-22$ was only partially suppressed by the late flowering ft-10, resulting in a phenotype of $f t-10$ spy-22 (17.4 \pm 1.4 $\mathrm{TRL})$ intermediate between $\mathrm{ft}-10(47.7 \pm 2.9 \mathrm{TRL})$ and the wild-type Col-0 (14.6 $\pm 1.3 \mathrm{TRL})$, while $\mathrm{ft}-10 \mathrm{sec}-5(46.2 \pm 6.4 \mathrm{TRL}$ ) was comparable to $\mathrm{ft}-10$ (Figure 1D, Table 1). This confirms previously shown results with mutants in the Ler-0 background (Tseng et al. 2004), indicating that SPY regulates flowering up- as well as down-stream from $F T$ and the photoperiod pathway, while SEC plays a minor role. This suggests that additional factors independent of the photoperiod pathway are affected by SPY. Thus, we also generated crosses with the late flowering Col-0 FRI. This line carries an active FRI allele introgressed from Sf-2, and consequently expresses high levels of the floral repressor FLOWERING LOCUS C (FLC) leading to very low levels of $F T$ expression and requirement of vernalization for floral induction (Clarke and Dean 1994; Lee and Amasino 1995). While late flowering of Col-0 FRI (74.2 \pm 7.8 $\mathrm{TRL}$ ) was not affected by sec-5 (data not shown), flowering is strongly accelerated in spy-22 $\mathrm{FRI}((26.0 \pm 1.4 \mathrm{TRL})$ (Figure 1E, Table 1). Levels of $F L C$ expression were maintained high in that line, while $F T$ was de-repressed (Figure 1F), suggesting that SPY represses flowering in Col-0 FRI independently of the vernalization pathway and FT. Thus, our data indicate that SPY represses floral transition only partly via FT and the photoperiod pathway, and spy-22 bypasses the vernalization requirement of Col-0 FRI.

\section{Mutants in SPY show accelerated transition from juvenile to adult phase}

Bypassing of the vernalization requirement of Col-0 FRI has previously been shown in lines expressing a miRNA-resistant stabilized version of SQUAMOSA promoter-binding protein-like 3 (rSPL3), that lacks a miRNA-binding site (Wang et al. 2009). SPL-transcription factors regulate the juvenile to adult phase transition as well as flowering time, and thus we analyzed also the juvenile to adult phase change in O-glycosylation mutants. This transition 
is morphologically marked by the formation of trichomes on the abaxial side of leaves and changes in leaf morphology (Telfer et al. 1997). We observed that spy-22 consistently transitioned early from the juvenile to the adult stage at $3.5 \pm 0.6$ juvenile leaves $(\mathrm{JL})$ compared to $5.8 \pm 0.6 \mathrm{JL}$ in Col-0, while sec $-5(5.9 \pm 0.7 \mathrm{JL})$ did not show a significant difference to the wild-type (Figure 2A, Table 2). In order to dissect the juvenile to adult phase transition from floral transition, we included ft-10-spy-22 in this analysis. We found that $\mathrm{ft}-10$ had a delayed juvenile-to adult transition, with $9.2 \pm 1.1 \mathrm{JL}$, which had been shown before (Willmann and Poethig 2011). On the other hand, $f t-10$ spy-22 was indistinguishable from spy-22 with $3.5 \pm$ $0.6 \mathrm{JL}$ (Figure 2B, Table 2), even though ft-10 spy-22 showed an extended adult vegetative phase and was flowering considerably later than spy-22 (Figure 1D, Table 1). This suggests that phase transition is regulated independently of daylength-dependent flowering time in spy22. Juvenile to adult transition is regulated by an internal developmental program, orchestrated by a balance of counteracting miR156 and miR172 (Wu and Poethig 2006; Wu et al. 2009). Thus, we analyzed transcript levels of primary miRNA156a, miRNA156b and miRNA156c in 5 day old seedlings, but did not find differences between wild-type and spy-22 (Figure 2C).

Levels of miR156 are, among other pathways, regulated by the accumulation of sugars in the leaves of growing plants (Yang et al. 2013; Yu et al. 2013). Cellular nutrient availability and especially increased sugar levels, enhance global levels of O-glycosylation in animals (Walgren et al. 2003; Hart 2014; Olivier-Van Stichelen et al. 2014; Peng et al. 2017). To test if O-glycosylation might be involved in regulation of miR156 abundance in response to sugar levels also in plants, we treated 5-day old Col-0, spy-22 and sec-5 seedlings with $50 \mathrm{mM}$ sucrose. While we could reproduce the results of a strong decrease in miR156a, miR156b and miR156c in response to sucrose treatment shown before (Yang et al. 2013; Yu et al. 2013), we did not see any differences between our mutant lines and wild-type (Figure 2D). Taken together, spy-22 showed accelerated phase change, but our results do not show an involvement of O-fucosylation in regulating the levels or balance of miR156 and miR172 during developmental phase transitions.

\section{SPY regulates phase transitions via SPL transcription factors independently of miRNA156}

Next we wanted to further test for a potential interaction between miRNA156 and Oglycosylation in the regulation of developmental transitions and generated 35S::MIRNA156a lines in Col-0, spy-22 and sec-5. Overexpression of miRNA156a in Col-0 lead to a strong delay of the juvenile to adult phase transition and extremely delayed flowering, as shown before (Wang et al. 2009; Wu et al. 2009). On the other hand, late flowering of this construct was strongly suppressed in spy-22, and to a smaller extent also in sec-5 (Figure 2E, Fig.S2) indicating that both O-fucosylation and O-GIcNAcylation repress flowering downstream of miR156.

miR156 targets the family of SPL-transcription factors, which induce phase transitions and floral transition (Wu and Poethig 2006; Wu et al. 2009; Xu et al. 2016; He et al. 2018). We therefore generated crosses of the O-glycosylation mutants with sp/9-4 sp/15-1, a line displaying delayed transitions, to test if SPLs are necessary for early transitions of spy-22. We included SPL9::rSPL9:GFP (Wang et al. 2009) in our analysis, a line carrying a miRNA resistant version of SPL9 that consequently displays very early phase transitions. In long photoperiods, early juvenile to adult transition of spy-22 (3.5 $\pm 0.6 \mathrm{JL})$ was slightly suppressed in the spy sp/9/15 triple cross $(5.4 \pm 0.7 \mathrm{JL})$, and flowering time was not strongly affected $(7.5$ 
$\pm 0.9 \mathrm{TRL}$ in spy-22 and 10.2 $\pm 1.3 \mathrm{TRL}$ in spy sp/9/15, Figure 3A, C and F, Table 3). This can be explained by the effect of SPY on the photoperiod pathway and FT expression, which function independently and in parallel to the aging pathway. Interestingly, in the sec sp/9/15 triple cross, the number of juvenile leaves $(6.8 \pm 0.7 \mathrm{JL})$ was unchanged compared to sec-5 (5.9 $\pm 0.7 \mathrm{JL})$, but sec-5 slightly accelerated the late flowering of $s p / 9-4 / 15-1$ (20.4 $\pm 1.7 \mathrm{TRL}$ in sp/9-4/15-1, and 16.4 $\pm 1.7 \mathrm{TRL}$ in sec sp/9/15) indicating that SPY and SEC might have common functions in this pathway (Figure $3 \mathrm{~A}, \mathrm{C}$ and $\mathrm{F}$, Table 3). In short photoperiods, early flowering of spy-22 (16.4 $\pm 3.6 \mathrm{TRL})$ is strongly suppressed in spy sp/9/15 (40.3 $\pm 4.7 \mathrm{TRL})$ while the juvenile to adult transition was only slightly affected $(5.1 \pm 0.8 \mathrm{JL}$ in spy-22 and 7.0 $\pm 1.1 \mathrm{JL}$ in spy sp/9/15, Figure 3B, D and F, Table 3). This suggests that early flowering of spy-22 in short photoperiods depends on functional SPL9 and/or SPL15, while the juvenile to adult transition is accelerated in $s p y-22$ by other factors, potentially other SPLs. We did not see a full suppression of the early flowering of $s p y-22$ in spy sp/9/15 to the level of sp/9-4/15$1(68.0 \pm 7.5 \mathrm{TRL})$, which might be explained by the fact that the SPLs are encoded by a gene family of 15 members with partially overlapping functions (Xing et al. 2010; Xu et al. 2016), and potentially higher order SPL-mutants could lead to a stronger effect. Surprisingly, flowering time of sec sp/9/15 could not be determined, as the primary inflorescences did not bolt, but multiple axillary meristems developed and went into flowering, making the leaf counting inaccurate (Figure 3D and $\mathrm{E}$ ), an effect we also frequently observed in sec- $5 \mathrm{FRI}$ and ft-10 sec-5 (not shown). In contrast to spy sp/9/15, the juvenile-to-adult transition of $s p / 9 / 15$ $(22.4 \pm 1.3 \mathrm{JL})$ was only slightly accelerated in sec sp/9/15 (18.0 $\pm 1.3 \mathrm{JL})$ suggesting that the effect of SEC is weaker than the one of SPY in this context. We also observed leaf growth rates in parallel to determining leaf numbers (Figure $3 \mathrm{G}$ ). SPL9::rSPL9:GFP was previously described as having a long plastochron, producing fewer leaves per day than wildtype, while sp/9-4/15-1 has a short plastochron (Wang et al. 2008). The slow leaf growth rate in spy-22 was comparable to SPL9::rSPL9:GFP (Figure 3G), which was completely suppressed in spy $s p / 9 / 15$ (Figure 3G, left panel). The leaf growth rate of sec-5 was also lower than that of Col0 , but to a far lesser extent, and again this phenotype was suppressed in sec sp/9/15 (Figure $3 G$, right panel). Together with the results seen in sec-5 35S::MIRNA156a, this suggests that SPY and SEC both negatively regulate phase change and flowering via SPLs, but independently of miR156, and SPY has a much stronger impact than SEC.

\section{SPY O-fucosylates SPL transcription factors}

Several SPLs were recently shown to be O-GIcNAc modified, among them also SPL8, which does not carry a miR156 recognition site (Xu et al. 2017). We therefore tested if SPLS that are important for the control of flowering time interact directly with SPY and can be Ofucosylated. 35S::Flag:SPY was co-infiltrated with 35S::SPL15:HA and 35S::SPL8:HA as a positive control, respectively, in Nicotiana benthamiana leaves for co-immunoprecipitation. When analyzing protein expression after infiltration of Nicotiana benthamiana leaves, we could clearly see a shift of SPL8 and SPL15 when co-infiltrated with SPY compared to the independent infiltration, suggesting a modification of SPL8 and SPL15 by SPY (Figure 4A). Interestingly we also consistently saw a stabilization of SPL8 and SPL15 in the samples coinfiltrated with SPY compared to single infiltrations (Figure 4, a representative example of several biological repeats is shown.) When precipitating 35S::Flag:SPY with an anti-Flag antibody, we could pull down SPL15, indicating protein-protein interaction (Figure 4B). These results indicate that SPY directly interacts with, and glycosylates SPLs. Together with our data from genetic analysis, this suggests that SPY inhibits SPL-activity by glycosylation. 


\section{DISCUSSION}

A multitude of different post-translational modifications (PTMs) confer complexity to the regulation of protein function and stability. They are essential components of signalling pathways in the course of development, often integrating environmental changes or finetuning crosstalk between different regulatory pathways. O-glycosylation of cytosolic and nuclear proteins is the modification of a number of very diverse proteins with a single monosaccharide on serine or threonine residues - which is in contrast to N-glycosylation events that involve the formation of branched carbohydrate chains of varying composition in the secretory pathway (Strasser 2016). Most organisms carry only one type of cytosolic Oglycosylation, with O-GlcNAcylation being the most common and best described example that is very well conserved among all kingdoms. Yeast is lacking an O-GlcNAc transferase, but uses O-mannose modification for the same molecular function instead, with many of the target proteins conserved between yeast and animal cells (Halim et al. 2015; Bandini et al. 2016), suggesting that in general the molecular function is more conserved than the type of monosaccharide involved. Plants are exceptional in that they use two different sugars attached to the same protein targets (Zentella et al. 2017). Currently the only other organism described to use fucosylation as well as GIcNAc modification is Toxoplasma gondii, but probably with distinct targets for the two different modifications (Bandini et al. 2016).

In Arabidopsis, only few O-glycosylated targets have been characterized in detail. The interaction of SPY with the bHLH transcription factors TCP14 and TCP15 in cytokinin response has been established (Steiner et al. 2012; Steiner et al. 2016) and SPY was also implicated in the integration of reactive oxygen species signalling during root development (Cui et al. 2014). Further protein interactions between SPY and GIGANTEA (Tseng et al. 2004), and SPY and SWI3C (Sarnowska et al. 2013) have been identified. However, by far the best characterised O-glycosylated protein in plants is the DELLA protein RGA. The current model suggests that O-GICNAc and O-fucose modification have opposite effects on DELLAs during gibberellin signalling, with O-GIcNAcylation leading to a closed conformation of DELLAs, rendering them less active. On the other hand fucosylation leads to an open conformation of RGA, facilitating the interaction with target transcription factors, thus increasing DELLA activity (Zentella et al. 2016; Zentella et al. 2017). However, a number of open questions remain, even in the context of gibberellin signalling, such as the fact that the double knockout of SPY and SEC is embryonic lethal even using weaker alleles, while the single mutants don't show drastic developmental phenotypes (Hartweck et al. 2006). This suggests that there might be targets where both modifications have the same effect, or even both modifications are necessary at the same time. A proteomics study using lectin weak affinity chromatography (LWAC) with glucosamine-binding wheat germ agglutinin (WGA) revealed O-GIcNAc modification of many different proteins, among them a number of transcriptional regulators, including SPLs (Xu et al. 2017). SPL9 and SPL15 directly interact with RGA (Yu et al. 2012; Yamaguchi et al. 2014; Hyun et al. 2016), and this interaction is likely to be affected by glycosylation of RGA as it was shown for other transcription factors such as BZR1, JAZ1, PIF3 and PIF4 (Zentella et al. 2016; Zentella et al. 2017). Here, we show that SPL15 additionally directly interacts with and is modified by SPY, suggesting that there is an additional, potentially independent effect of glycosylation on SPL15 function.

Our data suggest that the activity of SPLs is stabilized in both O-glycosylation mutants independently of miR156 in the miR156a overexpression lines, which is supported by the genetic analysis using sp/9/15 crosses with spy-22 and sec-5. It has previously been shown that miR156 overexpression lines are less sensitive to gibberellin treatment in terms of flowering time, keeping their extreme late flowering phenotype even when sprayed with 
gibberellin (Yu et al. 2012) (Wang et al. 2008). spy-mutants are often described as showing constitutive gibberellin signalling, but in contrast to gibberellin treatment of miR156 overexpression lines, we see a strong suppression of late flowering in spy-22 35S::MIR156a (Figure 2C). Moreover, albeit SPY and SEC have opposite effects in gibberellin signalling (Zentella et al. 2016; Zentella et al. 2017) sec-5 is also suppressing the late flowering of 35S::MIR156a (Figure 2C), and to a lower extent developmental transitions in long and short photoperiods (Figure $3 \mathrm{~A}-\mathrm{G}$ ), suggesting that the effect of glycosylation on SPLs might be independent of gibberellin signalling and DELLAs, as well as miR156, with a degree of redundancy between O-GIcNAc and O-fucose.

Overall, we suggest a model, where O-glycosylation regulates developmental transitions on multiple levels (Figure 4C). In long photoperiods, SPY suppresses expression of FT, via interaction with GI (Tseng et al. 2004). Additionally, glycosylation of RGA regulates its interaction with transcription factors regulating flowering time, such as PIFs (de Lucas et al. 2008; Kumar et al. 2012; Zentella et al. 2017) and probably also SPLs (Yu et al. 2012; Yamaguchi et al. 2014; Hyun et al. 2016). Our data add an additional level of regulation by direct modification of SPLs, independent of miR156 and potentially also independent of gibberellin.

\section{MATERIAL AND METHODS}

\section{Plant Material and growth conditions}

Arabidopsis thaliana ecotype Col-0 of was used as wild type. Lines spy-22 (SALK_090582), sec-5 (SALK_034290), sp/9-4 spl15-1 (N67865, (Schwarz et al. 2008)), ft-10 (GK-290E08, (Yoo et al. 2005)), FRI SF-2 (N6209) and pSPL9::GFP-rSPL9 (N9954, (Wang et al. 2009)), all in Col-0 background, were obtained from the European Arabidopsis Stock Centre (NASC) (Scholl et al., 2000). Seeds were surface sterilized with $70 \%$ ethanol and transferred $1 / 2$ Murashige and Skoog medium (2.15 g/L MS Salts, $0.25 \mathrm{~g} / \mathrm{L} \mathrm{MES,} \mathrm{pH} \mathrm{5.7,} \mathrm{1 \%} \mathrm{agar).} \mathrm{Seeds}$ were stratified in the dark at $4^{\circ} \mathrm{C}$ for 48 hours. Based upon the experiment the seedlings were germinated and grown in either long day (LD, 16 hours light / 8 hours dark) or short day (SD, 8 hours light / 16 hours dark) conditions at $22^{\circ} \mathrm{C}$. For studying phase change and flowering time, seedlings were transferred to soil at 5 days after germination.

\section{Flowering time and phase transition quantification}

Flowering time was quantified by counting the total number of rosette leaves (TRL) produced. Phase transitions were studied by observing the appearance of abaxial trichomes at the lower side of rosette leaves. Rosette leaves without any abaxial trichomes were grouped as juvenile leaves $(\mathrm{JL})$ and rosette leaves with abaxial trichomes were considered adult. Arabidopsis rosettes were harvested and individual leaves were taped to white paper and scanned for representations. Scanned pictures were edited to black and white using Paint 3D.

\section{RNA extraction and qPCR}

For expression analysis, seedlings were grown on $1 / 2$ MS plates. For studying the primary miRNA expression in response to sucrose treatment, sterilized seeds were germinated in 50 $\mathrm{ml} 1 / 2 \mathrm{MS}$ liquid media with shaking at $140 \mathrm{rpm}$, and seedlings were transferred to $1 / 2 \mathrm{MS}$ media supplemented with and without $50 \mathrm{mM}$ sucrose, and seedlings were harvested after $24 \mathrm{~h} .200$ mg of total plant material was used for total RNA extraction using SV Total Isolation System 
(Promega). $1 \mu \mathrm{g}$ of RNA was used for cDNA synthesis with the iScript ${ }^{\mathrm{TM}} \mathrm{cDNA}$ Synthesis kit (Bio-Rad). GoTaq ${ }^{\circledR}$ qPCR Master Mix (Promega) was used for quantitative real-time PCR, primers are listed in Table 4 and data was analyzed with Bio-Rad CFX Manager and Microsoft Excel for relative quantification using the 2(-DeltaDelta C(T))-method (Livak and Schmittgen 2001). For technical repeats, every sample was done in triplicates, representative results from one of at least two biological replicates are shown (as given in the figure legends).

\section{Plasmid construction and generation of transgenic lines}

miR156a overexpressing and SPY::SPY:Flag constructs were generated by amplifying the genomic DNA from Col-0, and for transient overexpression, SPY, SPL8 and SPL15 were amplified from cDNA to generate the respective tagged constructs using Q5 high fidelity DNA polymerase (NEB). The primers (listed in Table 4) contained 5'-overhangs binding to the linearized, Ncol / Xhol-digested backbone of the cloning vector pENTR ${ }^{\text {TM }} 4$. PCR products were excised and purified from agarose gel using GeneJET Gel Extraction Kit (Thermo Fisher) and cloned into Gateway ${ }^{\mathrm{TM}}$ pENTR ${ }^{\mathrm{TM}} 4$ by mixing the linearized vector backbone and PCR product in a 1:1 ratio using Gibson assembly (NEB), before transformation into $\mathrm{DH} 10 \mathrm{~B}$ electrocompetent $E$. coli cells. Plasmids containing the gene of interest were extracted using GeneJET Plasmid Miniprep Kit (Thermo Fisher) and confirmed by sequencing.

Plant expression vectors were generated using the above created entry clones and destination vectors pK7WG2D (Karimi et al., 2002) for 35S::MIR156a, pEarleyGate202 for 35S::Flag:SPY, pEarleyGate201 for 35S::HA:SPL8 or 35S::HA:SPL15 and pEarleyGate302 for SPY::SPY:Flag (Earley et al., 2006). Recombination of the entry clones with the destination vectors was done using Gateway LR Clonase II enzyme mix. Positive colonies with the plasmid of interest were selected for spectinomycin $(150 \mu \mathrm{g} / \mathrm{mL})$ resistance for miRNA overexpressing pK7WG2D constructs, and kanamycin $(50 \mu \mathrm{g} / \mathrm{mL})$ resistance for pEarleyGate201, pEarleyGate202 and pEarleyGate302 constructs, respectively on LB medium. Plasmids carrying the gene of interest were extracted from overnight bacterial culture using GeneJET Plasmid Miniprep Kit (Thermo Fisher) and confirmed by sequencing. Correct plasmids were transformed to Agrobacterium tumefaciens strain GV3101 (pMP90) before transformation of plants by floral dipping (Clough and Bent 1998). 35S:MIR156a constructs were transformed to Col-0, spy-22 and sec-5 backgrounds and SPY::SPY:Flag was introduced into spy-22 background.

\section{Transient protein expression in Nicotiana benthamiana}

For transient expression in Nicotiana benthamiana, Agrobacterium tumefaciens strain, GV3101 (pMP90) expressing 35S::Flag:SPY and 35S::HA:SPL8 or -SPL15 constructs were cultivated overnight using rifampicin $(50 \mu \mathrm{g} / \mathrm{mL})$, gentamycin $(50 \mu \mathrm{g} / \mathrm{mL})$ and kanamycin $(50 \mu \mathrm{g} / \mathrm{mL})$ selection. Bacterial cells were harvested, washed and resuspended in infiltration medium (500 mM MES, $20 \mathrm{mM} \mathrm{Na}_{3} \mathrm{PO}_{4}, 1 \mathrm{M}$ acetosyringone, $50 \mathrm{mg} / \mathrm{L} \mathrm{D-glucose)}$ to OD600 of 0.5 . For co-infiltration, equal amounts of the respective cultures were mixed. After infiltration on the abaxial side of a leaf of a five-week old $N$. benthamiana plant, the plants were maintained in the growth chamber for 3 days before harvesting.

\section{Protein extraction and co-immunoprecipitation}

For protein extraction, $1 \mathrm{~g}$ of agro-infiltrated leaves were frozen in liquid nitrogen, ground and taken up in $50 \mathrm{mM}$ Tris- $\mathrm{HCl} \mathrm{pH} \mathrm{7.6,150} \mathrm{mM} \mathrm{NaCl,} \mathrm{1 \%} \mathrm{Triton} \mathrm{X-100,} \mathrm{1x} \mathrm{plant} \mathrm{protease} \mathrm{inhibitor}$ cocktail (Sigma) and $20 \mu \mathrm{M}$ PUGNAc in the ratio 2:1. 100 $\mu \mathrm{L}$ extract was stored for further analysis as input. $50 \mu \mathrm{L}$ of anti-FLAG beads (Miltenyi Biotec) were added to the remaining 
plant extract and the samples were incubated on a rotor at $4^{\circ} \mathrm{C}$ for 30 minutes. A $\mu$ MACS column was placed in the magnetic field of a $\mu$ MACS separator and washed with above mentioned $200 \mu \mathrm{L}$ extraction buffer. Plant extract containing the anti-FLAG beads was added to the column and $100 \mu \mathrm{L}$ of the flow-through were collected and saved for further analysis. The column was rinsed 4 times with $200 \mu \mathrm{L}$ TBS $(50 \mathrm{mM}$ Tris-HCl pH 7.5, $150 \mathrm{mM} \mathrm{NaCl}, 1 \mathrm{x}$ plant protease inhibitor cocktail (Sigma), $20 \mu \mathrm{M}$ PuGNAc, $1.5 \mathrm{mM}$ DTE) and once with $100 \mu \mathrm{L}$ wash buffer $(20 \mathrm{mM}$ Tris- $\mathrm{HCl} \mathrm{pH} 7.5)$. For elution, $20 \mu \mathrm{L} 0.1 \mathrm{M}$ glycine, $\mathrm{pH} 2.3$ was loaded onto to the column and incubated for 3-5 minutes. After adding $60 \mu \mathrm{L} 0.1 \mathrm{M}$ glycine the first eluate was collected and neutralized with $20 \mu \mathrm{L} 0.5 \mathrm{M}$ Tris, pH 8.0. In the next step $20 \mu \mathrm{L}$ of preheated $95^{\circ} \mathrm{C}$ hot Laemmli buffer $(10 \%$ glycerol, $60 \mathrm{mM}$ Tris- $\mathrm{HCl} \mathrm{pH} 6.8,2 \%$ SDS, $0.1 \%$ bromophenol blue, $5 \% \beta$-mercaptoethanol) was loaded to the column and incubated for 5 minutes. Subsequently, $80 \mu \mathrm{L}$ of pre-heated $95^{\circ} \mathrm{C}$ hot Laemmli buffer were added and the eluate was collected and used for SDS-PAGE analysis and Western blotting.

\section{Western Blotting and antibody dilutions}

Input samples and eluates from the co-immunoprecipitation from both individual and coinfiltrated samples were subjected to SDS PAGE, and transferred to a PVDF membrane (Roth Roti®-PVDF, pore size $0.45 \mu \mathrm{m}$ ) using the wet transfer method in the Mini-Protean TetraSystem (Bio-Rad). The membranes were washed with PBST (137 mM NaCl, $2.7 \mathrm{mM} \mathrm{KCl}, 10$ $\mathrm{mM} \mathrm{Na}_{2} \mathrm{HPO}_{4}, 1.8 \mathrm{mM} \mathrm{KH}_{2} \mathrm{PO}_{4}, 0.1 \%(\mathrm{v} / \mathrm{v})$ Tween 80) for 10 minutes, blocked with $3 \%$ milk for 1 hour at room temperature and probed with anti-FLAG M2 monoclonal antibody (mouse, Sigma F1804, 1:2000 in 3\% milk/PBST), or blocked with 5\% BSA for 1 hour at room temperature and probed with anti-HA antibody (rabbit, Cell Signaling Technology 3742S, $1: 1000$ in $5 \%$ BSA/PBST) respectively. After probing at $4^{\circ} \mathrm{C}$ overnight, the membranes were washed with PBST before incubation with the respective secondary antibodies, goat antimouse HRP (1:10000) (Dianova 115-035-164), and goat anti-rabbit HRP (1:20000, Agrisera A S09 602) for 1 hour at room temperature. After washing, Bio-Rad Clarity Western ECL substrate was used for chemiluminescence detection on a Fusion Solo S (Vilber).

\section{Data analysis}

We used Excel for analysis of gene expression, and GraphPad Prism 5 and R (ggplot2, R Core Team, https://www.r-project.org/) for statistical analysis and generating graphs for quantification of rosette leaves. In the leaf quantification graphs, the mean is shown and error bars represent standard deviation, $\mathrm{n}$ is given in the respective tables. For statistical analysis, one-way ANOVA and Tukey's multiple comparison test were done.

\section{ACKNOWLEDGEMENTS}

We gratefully acknowledge Marie-Theres Hauser for sharing equipment, Lukas Grinninger for technical support and Julia König for supplying Nicotiana benthamiana plants. We thank Barbara Korbei, Vinod Kumar and Jürgen Kleine-Vehn for valuable input, and Mischa Lucyshyn for training and support with R. This work was supported by the Austrian Academy of Sciences ÖAW (DOC-fellowship to K.V.M. and APART fellowship to D.L.) and the Austrian Science Fund FWF (P-29051).

\section{AUTHOR CONTRIBUTIONS:}

D.L. conceived the project, D.L. and K.V.M designed experiments and wrote the manuscript. K.V.M. performed most of the experiments with the help of N.N., C.M. and I.Z. 


\section{REFERENCES}

Achard P, Herr A, Baulcombe DC, Harberd NP. 2004. Modulation of floral development by a gibberellin-regulated microRNA. Development 131: 3357-3365.

Andres F, Coupland G. 2012. The genetic basis of flowering responses to seasonal cues. Nat Rev Genet 13: 627-639.

Bandini G, Haserick JR, Motari E, Ouologuem DT, Lourido S, Roos DS, Costello CE, Robbins PW, Samuelson J. 2016. O-fucosylated glycoproteins form assemblies in close proximity to the nuclear pore complexes of Toxoplasma gondii. Proceedings of the National Academy of Sciences of the United States of America 113: 11567-11572.

Baurle I, Dean C. 2006. The timing of developmental transitions in plants. Cell 125: 655-664.

Clarke JH, Dean C. 1994. Mapping FRI, a locus controlling flowering time and vernalization response in Arabidopsis thaliana. Molecular \& general genetics : MGG 242: 81-89.

Clough SJ, Bent AF. 1998. Floral dip: a simplified method for Agrobacterium-mediated transformation of Arabidopsis thaliana. The Plant journal : for cell and molecular biology 16: 735-743.

Cui H, Kong D, Wei P, Hao Y, Torii KU, Lee JS, Li J. 2014. SPINDLY, ERECTA, and Its Ligand STOMAGEN Have a Role in Redox-Mediated Cortex Proliferation in the Arabidopsis Root. Molecular plant.

de Lucas M, Daviere JM, Rodriguez-Falcon M, Pontin M, Iglesias-Pedraz JM, Lorrain S, Fankhauser C, Blazquez MA, Titarenko E, Prat S. 2008. A molecular framework for light and gibberellin control of cell elongation. Nature 451: 480-484.

Feng S, Martinez C, Gusmaroli G, Wang Y, Zhou J, Wang F, Chen L, Yu L, Iglesias-Pedraz JM, Kircher S et al. 2008. Coordinated regulation of Arabidopsis thaliana development by light and gibberellins. Nature 451: 475-479.

Fouracre JP, Poethig RS. 2016. The role of small RNAs in vegetative shoot development. Current opinion in plant biology 29: 64-72.

Galvao VC, Collani S, Horrer D, Schmid M. 2015. Gibberellic acid signaling is required for ambient temperature-mediated induction of flowering in Arabidopsis thaliana. The Plant journal : for cell and molecular biology 84: 949-962.

Halim A, Larsen IS, Neubert P, Joshi HJ, Petersen BL, Vakhrushev SY, Strahl S, Clausen H. 2015. Discovery of a nucleocytoplasmic O-mannose glycoproteome in yeast. Proceedings of the National Academy of Sciences of the United States of America 112: 15648-15653.

Harberd NP, Belfield E, Yasumura Y. 2009. The angiosperm gibberellin-GID1-DELLA growth regulatory mechanism: how an "inhibitor of an inhibitor" enables flexible response to fluctuating environments. The Plant cell 21: 1328-1339.

Hart GW. 2014. Nutrient Regulation of Cellular Metabolism \& Physiology by O-GlcNAcylation. Journal of Biological Chemistry.

Hartweck LM, Genger RK, Grey WM, Olszewski NE. 2006. SECRET AGENT and SPINDLY have overlapping roles in the development of Arabidopsis thaliana L. Heyn. Journal of Experimental Botany 57: 865-875.

He J, Xu M, Willmann MR, McCormick K, Hu T, Yang L, Starker CG, Voytas DF, Meyers BC, Poethig RS. 2018. Threshold-dependent repression of SPL gene expression by miR156/miR157 controls vegetative phase change in Arabidopsis thaliana. PLoS Genet 14: e1007337.

Huijser P, Schmid M. 2011. The control of developmental phase transitions in plants. Development 138: 4117-4129.

Hyun Y, Richter R, Vincent C, Martinez-Gallegos R, Porri A, Coupland G. 2016. Multi-layered Regulation of SPL15 and Cooperation with SOC1 Integrate Endogenous Flowering Pathways at the Arabidopsis Shoot Meristem. Dev Cell 37: 254-266.

Jaeger KE, Wigge PA. 2007. FT protein acts as a long-range signal in Arabidopsis. Current biology : $C B$ 17: $1050-1054$. 
Jung JH, Ju Y, Seo PJ, Lee JH, Park CM. 2012. The SOC1-SPL module integrates photoperiod and gibberellic acid signals to control flowering time in Arabidopsis. The Plant journal : for cell and molecular biology 69: 577-588.

Kumar SV, Lucyshyn D, Jaeger KE, Alos E, Alvey E, Harberd NP, Wigge PA. 2012. Transcription factor PIF4 controls the thermosensory activation of flowering. Nature 484: 242-245.

Lee I, Amasino RM. 1995. Effect of Vernalization, Photoperiod, and Light Quality on the Flowering Phenotype of Arabidopsis Plants Containing the FRIGIDA Gene. Plant Physiol 108: 157-162.

Li QF, Wang C, Jiang L, Li S, Sun SS, He JX. 2012. An interaction between BZR1 and DELLAs mediates direct signaling crosstalk between brassinosteroids and gibberellins in Arabidopsis. Science signaling 5: ra72.

Livak KJ, Schmittgen TD. 2001. Analysis of relative gene expression data using real-time quantitative PCR and the 2(-Delta Delta C(T)) Method. Methods 25: 402-408.

Mathieu J, Warthmann N, Kuttner F, Schmid M. 2007. Export of FT protein from phloem companion cells is sufficient for floral induction in Arabidopsis. Current biology : CB 17: 1055-1060.

Moon J, Suh SS, Lee H, Choi KR, Hong CB, Paek NC, Kim SG, Lee I. 2003. The SOC1 MADS-box gene integrates vernalization and gibberellin signals for flowering in Arabidopsis. The Plant journal : for cell and molecular biology 35: 613-623.

Olivier-Van Stichelen S, Dehennaut V, Buzy A, Zachayus J-L, Guinez C, Mir A-M, El Yazidi-Belkoura I, Copin M-C, Boureme D, Loyaux D et al. 2014. O-GlcNAcylation stabilizes $\beta$-catenin through direct competition with phosphorylation at threonine 41. The FASEB Journal 28: 3325-3338.

Peng C, Zhu Y, Zhang W, Liao Q, Chen Y, Zhao X, Guo Q, Shen P, Zhen B, Qian X et al. 2017. Regulation of the Hippo-YAP Pathway by Glucose Sensor O-GlcNAcylation. Mol Cell 68: 591604 e595.

Poethig RS. 2003. Phase change and the regulation of developmental timing in plants. Science 301: 334-336.

-. 2009. Small RNAs and developmental timing in plants. Curr Opin Genet Dev 19: 374-378.

Porri A, Torti S, Romera-Branchat M, Coupland G. 2012. Spatially distinct regulatory roles for gibberellins in the promotion of flowering of Arabidopsis under long photoperiods. Development 139: 2198-2209.

Reeves PH, Coupland G. 2001. Analysis of flowering time control in Arabidopsis by comparison of double and triple mutants. Plant Physiol 126: 1085-1091.

Sarnowska EA, Rolicka AT, Bucior E, Cwiek P, Tohge T, Fernie AR, Jikumaru Y, Kamiya Y, Franzen R, Schmelzer E et al. 2013. DELLA-interacting SWI3C core subunit of switch/sucrose nonfermenting chromatin remodeling complex modulates gibberellin responses and hormonal cross talk in Arabidopsis. Plant Physiol 163: 305-317.

Schwarz S, Grande AV, Bujdoso N, Saedler H, Huijser P. 2008. The microRNA regulated SBP-box genes SPL9 and SPL15 control shoot maturation in Arabidopsis. Plant Mol Biol 67: 183-195.

Silverstone AL, Tseng T-S, Swain SM, Dill A, Jeong SY, Olszewski NE, Sun T-p. 2007. Functional Analysis of SPINDLY in Gibberellin Signaling in Arabidopsis. Plant Physiology 143: 987-1000.

Steiner E, Efroni I, Gopalraj M, Saathoff K, Tseng TS, Kieffer M, Eshed Y, Olszewski N, Weiss D. 2012. The Arabidopsis O-linked N-acetylglucosamine transferase SPINDLY interacts with class I TCPs to facilitate cytokinin responses in leaves and flowers. The Plant cell 24: 96-108.

Steiner E, Livne S, Kobinson-Katz T, Tal L, Pri-Tal O, Mosquna A, Tarkowska D, Mueller B, Tarkowski P, Weiss D. 2016. The Putative O-Linked N-Acetylglucosamine Transferase SPINDLY Inhibits Class I TCP Proteolysis to Promote Sensitivity to Cytokinin. Plant Physiol 171: 1485-1494.

Strasser R. 2016. Plant protein glycosylation. Glycobiology 26: 926-939.

Swain SM, Tseng T-s, Olszewski NE. 2001. Altered Expression of SPINDLY Affects Gibberellin Response and Plant Development. Plant Physiology 126: 1174-1185.

Telfer A, Bollman KM, Poethig RS. 1997. Phase change and the regulation of trichome distribution in Arabidopsis thaliana. Development 124: 645-654. 
Tseng T-S, Salomé PA, McClung CR, Olszewski NE. 2004. SPINDLY and GIGANTEA Interact and Act in Arabidopsis thaliana Pathways Involved in Light Responses, Flowering, and Rhythms in Cotyledon Movements. The Plant Cell Online 16: 1550-1563.

Tseng T-S, Swain SM, Olszewski NE. 2001. Ectopic Expression of the Tetratricopeptide Repeat Domain of SPINDLY Causes Defects in Gibberellin Response. Plant Physiology 126: 12501258.

Walgren JL, Vincent TS, Schey KL, Buse MG. 2003. High glucose and insulin promote O-GIcNAc modification of proteins, including alpha-tubulin. American journal of physiology Endocrinology and metabolism 284: E424-434.

Wang JW, Czech B, Weigel D. 2009. miR156-regulated SPL transcription factors define an endogenous flowering pathway in Arabidopsis thaliana. Cell 138: 738-749.

Wigge PA, Kim MC, Jaeger KE, Busch W, Schmid M, Lohmann JU, Weigel D. 2005. Integration of spatial and temporal information during floral induction in Arabidopsis. Science 309: 10561059.

Willmann MR, Poethig RS. 2011. The effect of the floral repressor FLC on the timing and progression of vegetative phase change in Arabidopsis. Development 138: 677-685.

Wilson RN, Heckman JW, Somerville CR. 1992. Gibberellin Is Required for Flowering in Arabidopsis thaliana under Short Days. Plant Physiol 100: 403-408.

Wu G, Park MY, Conway SR, Wang JW, Weigel D, Poethig RS. 2009. The sequential action of miR156 and miR172 regulates developmental timing in Arabidopsis. Cell 138: 750-759.

Wu G, Poethig RS. 2006. Temporal regulation of shoot development in Arabidopsis thaliana by miR156 and its target SPL3. Development 133: 3539-3547.

Xing L, Liu Y, Xu S, Xiao J, Wang B, Deng H, Lu Z, Xu Y, Chong K. 2018. Arabidopsis O-GlcNAc transferase SEC activates histone methyltransferase ATX1 to regulate flowering. EMBO J 37.

Xing S, Salinas M, Höhmann S, Berndtgen R, Huijser P. 2010. miR156-Targeted and Nontargeted SBPBox Transcription Factors Act in Concert to Secure Male Fertility in <em>Arabidopsis</em>. The Plant cell 22: 3935-3950.

Xu M, Hu T, Zhao J, Park MY, Earley KW, Wu G, Yang L, Poethig RS. 2016. Developmental Functions of miR156-Regulated SQUAMOSA PROMOTER BINDING PROTEIN-LIKE (SPL) Genes in Arabidopsis thaliana. PLoS Genet 12: e1006263.

Xu SL, Chalkley RJ, Maynard JC, Wang W, Ni W, Jiang X, Shin K, Cheng L, Savage D, Huhmer AF et al. 2017. Proteomic analysis reveals O-GIcNAc modification on proteins with key regulatory functions in Arabidopsis. Proceedings of the National Academy of Sciences of the United States of America 114: E1536-E1543.

Yamaguchi N, Winter CM, Wu MF, Kanno Y, Yamaguchi A, Seo M, Wagner D. 2014. Gibberellin acts positively then negatively to control onset of flower formation in Arabidopsis. Science 344: 638-641.

Yang L, Conway SR, Poethig RS. 2011. Vegetative phase change is mediated by a leaf-derived signal that represses the transcription of miR156. Development 138: 245-249.

Yang L, Xu M, Koo Y, He J, Poethig RS. 2013. Sugar promotes vegetative phase change in Arabidopsis thaliana by repressing the expression of MIR156A and MIR156C. eLife 2: e00260.

Yoo SK, Chung KS, Kim J, Lee JH, Hong SM, Yoo SJ, Yoo SY, Lee JS, Ahn JH. 2005. CONSTANS activates SUPPRESSOR OF OVEREXPRESSION OF CONSTANS 1 through FLOWERING LOCUS T to promote flowering in Arabidopsis. Plant Physiol 139: 770-778.

Yu S, Cao L, Zhou CM, Zhang TQ, Lian H, Sun Y, Wu J, Huang J, Wang G, Wang JW. 2013. Sugar is an endogenous cue for juvenile-to-adult phase transition in plants. elife 2: e00269.

Yu S, Galvao VC, Zhang YC, Horrer D, Zhang TQ, Hao YH, Feng YQ, Wang S, Schmid M, Wang JW. 2012. Gibberellin regulates the Arabidopsis floral transition through miR156-targeted SQUAMOSA promoter binding-like transcription factors. The Plant cell 24: 3320-3332.

Zentella R, Hu J, Hsieh WP, Matsumoto PA, Dawdy A, Barnhill B, Oldenhof H, Hartweck LM, Maitra S, Thomas SG et al. 2016. O-GIcNAcylation of master growth repressor DELLA by SECRET 
bioRxiv preprint doi: https://doi.org/10.1101/618744; this version posted April 25, 2019. The copyright holder for this preprint (which was

not certified by peer review) is the author/funder. All rights reserved. No reuse allowed without permission.

Mutanwad et al., 2019

AGENT modulates multiple signaling pathways in Arabidopsis. Genes \& development 30: 164-176.

Zentella R, Sui N, Barnhill B, Hsieh WP, Hu J, Shabanowitz J, Boyce M, Olszewski NE, Zhou P, Hunt DF et al. 2017. The Arabidopsis O-fucosyltransferase SPINDLY activates nuclear growth repressor DELLA. Nature chemical biology. 


\section{TABLES}

Table 1:Rosette leaf numbers for graphs shown in Figure 1

\begin{tabular}{|c|c|c|c|}
\hline & & Rosette leaves & $\mathbf{n}$ \\
\hline \multirow{3}{*}{ LD } & Col-0 & $13.1 \pm 1.1$ & 51 \\
\hline & spy-22a & $8.6 \pm 1.4$ & 46 \\
\hline & $\sec -5^{a}$ & $11.8 \pm 1.6$ & 49 \\
\hline \multirow{3}{*}{ SD } & Col-0 & $66.0 \pm 7.9$ & 6 \\
\hline & spy-22 & $22.7 \pm 0.5$ & 6 \\
\hline & sec-5 & $61.0 \pm 5.0$ & 6 \\
\hline \multirow{12}{*}{ LD } & & Rosette leaves & $\mathbf{n}$ \\
\hline & Col-0 & $14.6 \pm 1.3$ & 12 \\
\hline & $f t-10$ & $47.7 \pm 2.9$ & 9 \\
\hline & spy-22 & $7.5 \pm 0.7$ & 12 \\
\hline & sec-5 & $14.9 \pm 1.5$ & 10 \\
\hline & $f t-10$ spy-22 & $17.4 \pm 1.4$ & 11 \\
\hline & $f t-10$ sec -5 & $46.2 \pm 6.4$ & 8 \\
\hline & & Rosette leaves & $\mathrm{n}$ \\
\hline & Col-0 & $14.7 \pm 1.3$ & 13 \\
\hline & spy-22 & $7.1 \pm 0.7$ & 13 \\
\hline & FRI & $74.2 \pm 7.8$ & 12 \\
\hline & FRI spy-22 & $26.0 \pm 1.4$ & 10 \\
\hline
\end{tabular}

One-way ANOVA, Tukeys Multiple Comparison Test:

a significantly lower than Col-0 $\left.{ }^{* * *} p \leq 0.001\right)$

Table 2: Rosette leaf numbers for graphs shown in Figure 2

\begin{tabular}{|c|c|c|c|c|}
\hline \multirow{8}{*}{ LD } & & Juvenile leaves & Rosette leaves & $\mathbf{n}$ \\
\hline & Col-0 & $5.8 \pm 0.6$ & $12.5 \pm 1.3$ & 42 \\
\hline & $s p y-22^{a}$ & $3.5 \pm 0.6^{* \star *}$ & $5.7 \pm 0.9^{\star \star \star *}$ & 33 \\
\hline & $\sec -5^{b}$ & $5.9 \pm 0.7^{\mathrm{ns}}$ & $11.4 \pm 0.8^{*}$ & 31 \\
\hline & Col-0 & $5.1 \pm 0.7$ & n.d. & 22 \\
\hline & spy-22 & $3.9 \pm 0.6$ & n.d. & 19 \\
\hline & $f t-10$ & $9.2 \pm 1.1$ & n.d. & 20 \\
\hline & $f t-10$ spy-22 & $3.5 \pm 0.6$ & n.d. & 16 \\
\hline
\end{tabular}

One-way ANOVA, Tukeys Multiple Comparison Test:

a significantly lower than Col-0 $\left({ }^{* * *} p \leq 0.001\right)$

${ }^{b}$ significantly less rosette leaves than Col-0 ( $\left.{ }^{*} p \leq 0.05\right)$ 
bioRxiv preprint doi: https://doi.org/10.1101/618744; this version posted April 25, 2019. The copyright holder for this preprint (which was not certified by peer review) is the author/funder. All rights reserved. No reuse allowed without permission.

Mutanwad et al., 2019

Table 3: Rosette leaf numbers for graphs shown in Figure 3

\begin{tabular}{|c|c|c|c|c|}
\hline & & Juvenile leaves & Rosette leaves & $\mathrm{n}$ \\
\hline \multirow{7}{*}{ LD } & Col-0 & $5.8 \pm 0.6$ & $12.5 \pm 1.3$ & 42 \\
\hline & $s p y-22^{a}$ & $3.5 \pm 0.6$ & $7.5 \pm 0.9$ & 33 \\
\hline & $\sec -5^{b}$ & $5.9 \pm 0.7^{\mathrm{ns}}$ & $11.4 \pm 0.8^{*}$ & 31 \\
\hline & $s p / 9-4 / 15-1^{c}$ & $8.8 \pm 0.6$ & $20.4 \pm 1.7$ & 37 \\
\hline & spy-22 sp/9-4/15-1 d e & $5.4 \pm 0.7$ & $10.2 \pm 1.3$ & 42 \\
\hline & sec-5 sp/9-4/15-1 ef & $6.8 \pm 0.7$ & $16.4 \pm 1.7$ & 46 \\
\hline & SPL9::rSPL9:GFP & $1.3 \pm 0.5$ & $5.6 \pm 1.3$ & 37 \\
\hline & & Juvenile leaves & Rosette leaves & $\mathbf{n}$ \\
\hline \multirow{7}{*}{ SD } & Col-0 & $12.4 \pm 1.1$ & $55.3 \pm 4.7$ & 15 \\
\hline & $s p y-22^{a}$ & $5.1 \pm 0.8$ & $16.4 \pm 3.6$ & 15 \\
\hline & $\sec -5 \mathrm{~g}$ & $11.3 \pm 0.8^{* \star}$ & $50.1 \pm 5.3^{\mathrm{ns}}$ & 15 \\
\hline & $s p / 9-4 / 15-1^{c}$ & $22.4 \pm 1.3$ & $68.0 \pm 7.5$ & 14 \\
\hline & spy-22 sp/9-4/15-1 d e & $7.0 \pm 1.1$ & $40.3 \pm 4.7$ & 14 \\
\hline & sec-5 sp/9-4/15-1 fh & $18.0 \pm 1.3$ & n.d. & 14 \\
\hline & SPL9::rSPL9:GFP & $1.5 \pm 0.5$ & $43.7 \pm 3.7$ & 15 \\
\hline
\end{tabular}

One-way ANOVA, Tukeys Multiple Comparison Test:

a significantly less leaves than Col-0 (*** $p \leq 0.001)$

${ }^{b}$ significantly less rosette leaves than Col-0 (* $\left.p \leq 0.05\right)$

${ }^{\mathrm{c}}$ significantly more leaves than Col-0 ( $\left.{ }^{* * *} p \leq 0.001\right)$

${ }^{\mathrm{d}}$ significantly more leaves than spy-22 (*** $\left.p \leq 0.001\right)$

${ }^{e}$ significantly less leaves than $s p / 9-4 / 15-1\left({ }^{* * *} p \leq 0.001\right)$

${ }^{f}$ significantly more leaves than sec-5 $\left(^{* * *} p \leq 0.001\right)$

$\mathrm{g}$ significantly less juvenile leaves than Col-0 ( $\left.{ }^{* *} p \leq 0.01\right)$

${ }^{\mathrm{h}}$ significantly less juvenile leaves than $\left.s p / 9-4 / 15-1{ }^{* *} p \leq 0.01\right)$ 
bioRxiv preprint doi: https://doi.org/10.1101/618744; this version posted April 25, 2019. The copyright holder for this preprint (which was not certified by peer review) is the author/funder. All rights reserved. No reuse allowed without permission.

Mutanwad et al., 2019

Table 4: Oligonucleotides used in this study

\begin{tabular}{|c|c|c|c|}
\hline ID & Sequence & Orientation & Purpose \\
\hline \multirow{2}{*}{ 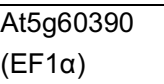 } & TGAGCACGCTCTTCTTGCTTTCA & forward & \multirow{2}{*}{ qPCR, reference } \\
\hline & GGTGGTGGCATCCATCTTGTTACA & reverse & \\
\hline \multirow{2}{*}{ pri-miR156a } & CTTCGTTCTCTATGTCTCAATCTCTC & forward & \multirow{2}{*}{$\begin{array}{c}\text { qPCR } \\
\text { (Yang et al., 2013) }\end{array}$} \\
\hline & TGATTAAAGGCTAAAGGTCTCCTC & reverse & \\
\hline \multirow{2}{*}{ pri-miR156b } & GTGATAATGAGTGATGACTGATG & forward & \multirow{2}{*}{$\begin{array}{c}\text { qPCR } \\
\text { (Yang et al., 2013) }\end{array}$} \\
\hline & GAAAACGTGACCGGGACCGAATCG & reverse & \\
\hline \multirow{2}{*}{ pri-miR156c } & GTGATAATGAGTGATGACTGATG & forward & \multirow{2}{*}{$\begin{array}{c}\text { qPCR } \\
\text { (Yang et al., 2013) }\end{array}$} \\
\hline & GAAAACGTGACCGGGACCGAATCG & reverse & \\
\hline \multirow{2}{*}{ FT } & CATTTTATGATACGAGTAACGAACGGTG & forward & \multirow{2}{*}{ qPCR } \\
\hline & САСТСТСАТТТТССТСССССТСТС & reverse & \\
\hline \multirow{2}{*}{ FLC } & TCATGTGGGAGCAGAAGCTG & forward & \multirow{2}{*}{ qPCR } \\
\hline & CCGCCGATTTAAGGTGGCTA & reverse & \\
\hline
\end{tabular}

\begin{tabular}{|c|c|c|c|}
\hline ID & Sequence & Orientation & Purpose \\
\hline \multirow{3}{*}{ spy-22 } & GTTAAACCCTAAGTATCGGAC & forward & \multirow{3}{*}{ Genotyping } \\
\hline & TTGGCATAAGAAAGTGTATC & reverse & \\
\hline & ATTTTGCCGATTTCGGAAC & T-DNA & \\
\hline \multirow{3}{*}{$\sec -5$} & CACGCCTGGCTCTTGCTCATCAG & forward & \multirow{3}{*}{ Genotyping } \\
\hline & GCGGATTGCACGATCAGTGTC & reverse & \\
\hline & ATTTTGCCGATTTCGGAAC & T-DNA & \\
\hline \multirow{3}{*}{ spl 15-1 } & TGTTGGTGTCTGAAGTTGCTG & forward & \multirow{3}{*}{ Genotyping } \\
\hline & TCCACCGAGTCTTCTTCACTC & reverse & \\
\hline & TGGTTCACGTAGTGGGCCATCG & T-DNA & \\
\hline \multirow{3}{*}{$s p / 9-4$} & TGGTTCCTCCACTGAGTCATC & forward & \multirow{3}{*}{ Genotyping } \\
\hline & GCTCATTATGACCAGCGAGTC & reverse & \\
\hline & TAGCATCTGAATTTCATAACCAATCTCGATACAC & T-DNA & \\
\hline \multirow{3}{*}{$\mathrm{ft}-10$} & TAAGCTCAATGATATTCCCGTACA & forward & \multirow{3}{*}{ Genotyping } \\
\hline & CAGGTTCAAAACAAGCCAAGA & T-DNA & \\
\hline & CCCATTTGGACGTGAATGTAGACAC & reverse & \\
\hline \multirow[b]{2}{*}{ Col-0 FRI } & ATGAGATTGCCGGTGCTTT & forward & \multirow{2}{*}{ Genotyping } \\
\hline & TGGTCGATGATGTCAACAAAA & reverse & \\
\hline
\end{tabular}

\begin{tabular}{|c|c|c|c|}
\hline ID & Sequence & Orientation & Purpose \\
\hline \multirow{2}{*}{ miRNA156 } & TACAAAAAAGCAGGCTCCACTCTTTGTCTTCTCCAGTTAAAAC & forward & \multirow{2}{*}{ Cloning 35::MIRNA156a } \\
\hline & GCTGGGTCTAGATATCTCGACAAGAGAGACAGAGAAAG & reverse & \\
\hline \multirow{4}{*}{ SPY } & TACAAAAAAGCAGGCTCCACAGACAAGAGGGTTTTATAACTC & forward & \multirow{2}{*}{$\begin{array}{c}\text { Cloning SPY-promotor + } \\
\text { Flag }\end{array}$} \\
\hline & TGTCGTCATCGTCTTTGTAGTCCATTTTTTTGTAACTAAAATCTTG & reverse & \\
\hline & CTACAAAGACGATGACGACAAGGTGGGACTGGAAGATGATAC & forward & \multirow[b]{2}{*}{ Cloning Flag + SPY-ORF } \\
\hline & GCTGGGTCTAGATATCTCGACTAGCTAGTGGAGTCCATTC & reverse & \\
\hline \multirow{2}{*}{ SPL8 } & TACAAAAAAGCAGGCTCCACCTCCTCCACCCCTTCCG & forward & \multirow{2}{*}{ Cloning $35 S:: H A: S P L 8$} \\
\hline & GCTGGGTCTAGATATCTCGATCCGCTGGAGAAAAACATTG & reverse & \\
\hline \multirow[b]{2}{*}{ SPL15 } & TACAAAAAAGCAGGCTCCACCACCATGGAGTTGTTAATGTGTTC & forward & \multirow{2}{*}{ Cloning $35 S:: H A: S P L 8$} \\
\hline & GCTGGGTCTAGATATCTCGATCAAAGAGACCAATTGAAATG & reverse & \\
\hline
\end{tabular}



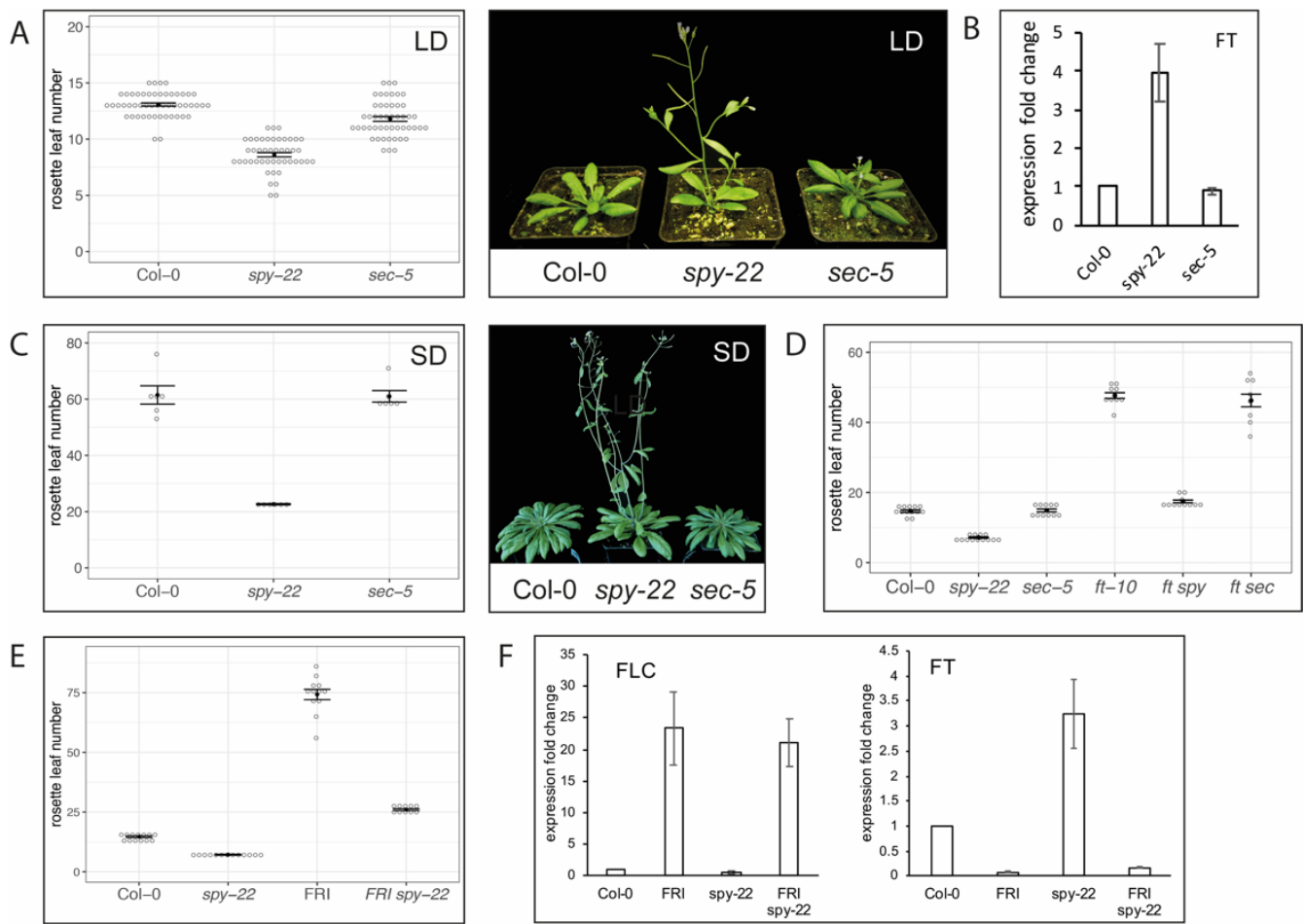

Figure 1. SPY suppresses flowering in long and short photoperiods.

(A) Total rosette leaf numbers of wildtype Col-0, spy-22 and sec-5 grown in LD conditions (left panel), and representative pictures (right panel). (B) Relative expression levels of $F T$ in 10 day old seedlings of spy-22 and sec-5 grown in LD conditions, an average of three biological repeats +/- SEM is shown, $\mathrm{n}>20$. (C) Total rosette leaf numbers of wildtype Col-0, spy-22 and sec-5 grown in SD conditions (left panel), and representative pictures (right panel). (D) Total rosette leaf numbers of wildtype Col-0, spy22, sec-5, ft-10, ft spy and ft sec grown in LD conditions. (E) Total rosette leaf numbers of wildtype Col0 , spy-22, Col-0 FRI and FRI spy-22 grown in LD conditions, and (F) relative expression levels of FLC and $F T$ in 10-day old seedlings of Col-0, spy-22, Col-0 FRI and FRI spy-22 grown in LD conditions.

For comparison of rosette leaf numbers, one-way ANOVA with Tukey's multiple comparison was done, $\mathrm{n}$ is given in Table $\left.1{ }^{* * *} \mathrm{p} \leq 0.001,{ }^{*} \mathrm{p} \leq 0.05\right)$.
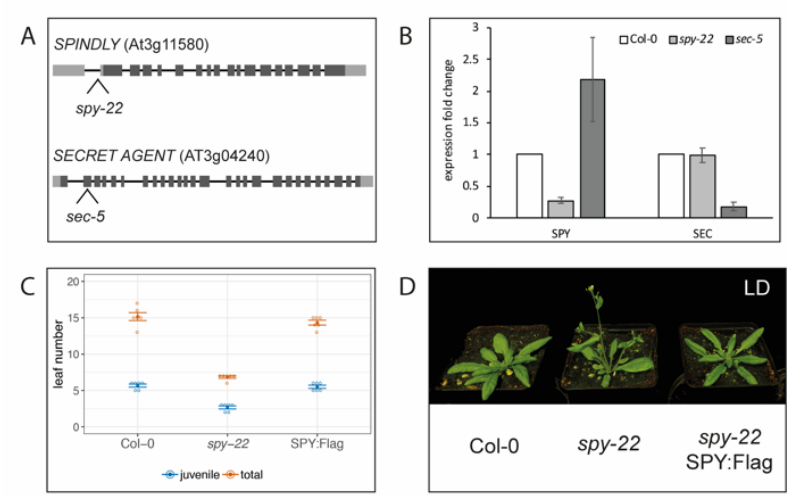

\section{Supplemental Figure 1. Description of mutant lines spy-22 and sec-5}

(A) Structure of SPY and SEC, 5'- and 3'-UTR are shown in light grey and exons in dark grey boxes, the lines represent introns. The positions of T-DNA insertion in spy-22 (SALK_090582) and sec-5 (SALK_034290) are given. (B) Relative expression levels of SPY and SEC in spy-22 and sec-5 seedlings, an average of three biological repeats + - SEM is shown, $n>20$. (C) Juvenile and total rosette leaf numbers of wildtype Col-0, spy-22, and spy-22 SPY::SPY:Flag (SPY:Flag) grown in LD conditions. (D) Representative pictures of Col-0, spy-22, and spy-22 SPY::SPY:Flag (SPY:Flag) grown in LD conditions. 

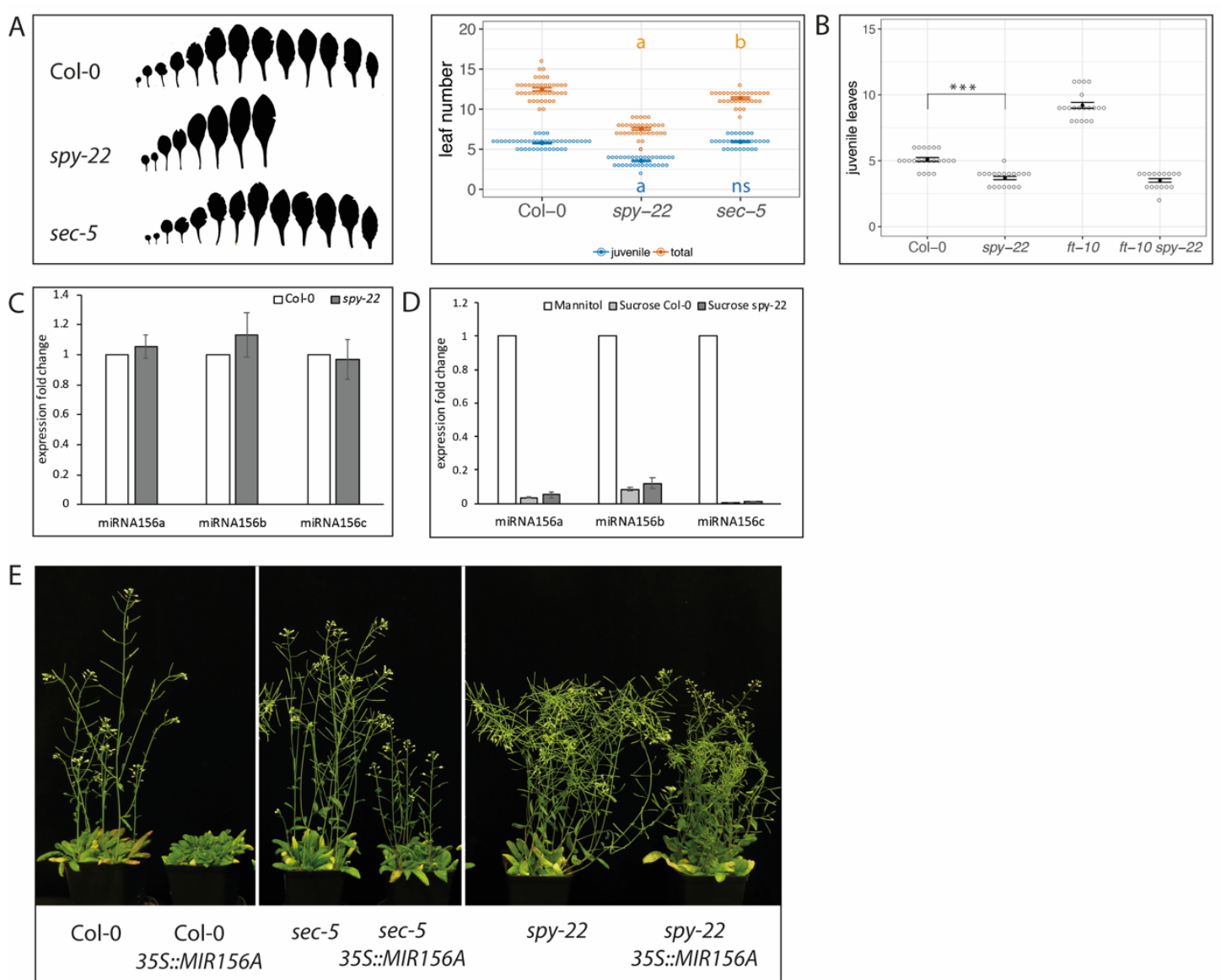

Figure 2. SPY regulates phase transitions independently of miR156.

(A) Leaf scans (left panel), and juvenile and total leaf numbers (right panel) of wildtype Col-0, spy-22 and sec-5 grown in LD conditions. ${ }^{a}$ significantly lower than Col-0 ( $\left.{ }^{* * *} p \leq 0.001\right),{ }^{b}$ significantly lower than Col-0 ( ${ }^{*} p \leq 0.05$ ), ns: not significant. (B) Juvenile leaf numbers of wildtype Col-0, spy-22, ft-10 and ft-10 spy-22 grown in LD conditions ( $\left.{ }^{* * *} p \leq 0.001\right)$. (C) Relative expression levels of miR156 in Col- 0 and spy-22 in 6 day old seedlings. (D) Relative expression levels of miR156 in 6 day old seedlings treated with $50 \mathrm{mM}$ sucrose for $24 \mathrm{~h}$. An average of two biological repeats, +/- SEM of all technical repeats is shown, $\mathrm{n}>20$. (E) Col-0, Col-0 35S::MIR156A, sec-5, sec-5 35S::MIR156A, spy-22, spy-22 35S::MIR156A, four plants per pot grown in LD conditions, representative pictures of 7 week old plants are shown.

For comparison of rosette leaf numbers, one-way ANOVA with Tukey's multiple comparison was done, $\mathrm{n}$ is given in Table 2 .

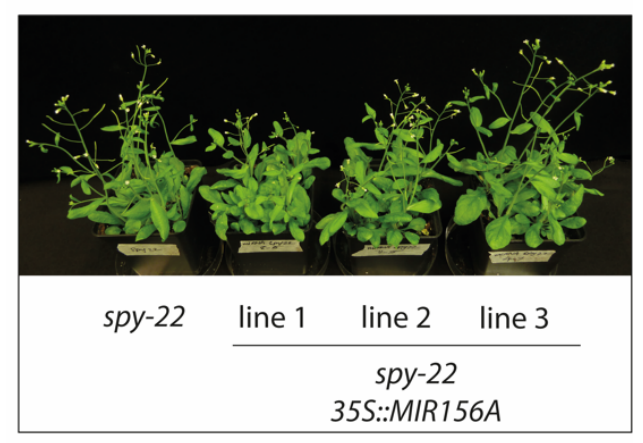

\section{Supplemental Figure 2.}

Three lines of independent transformants of spy-22 35S::MIR156A, 5 week old plants grown in LD conditions are shown. 

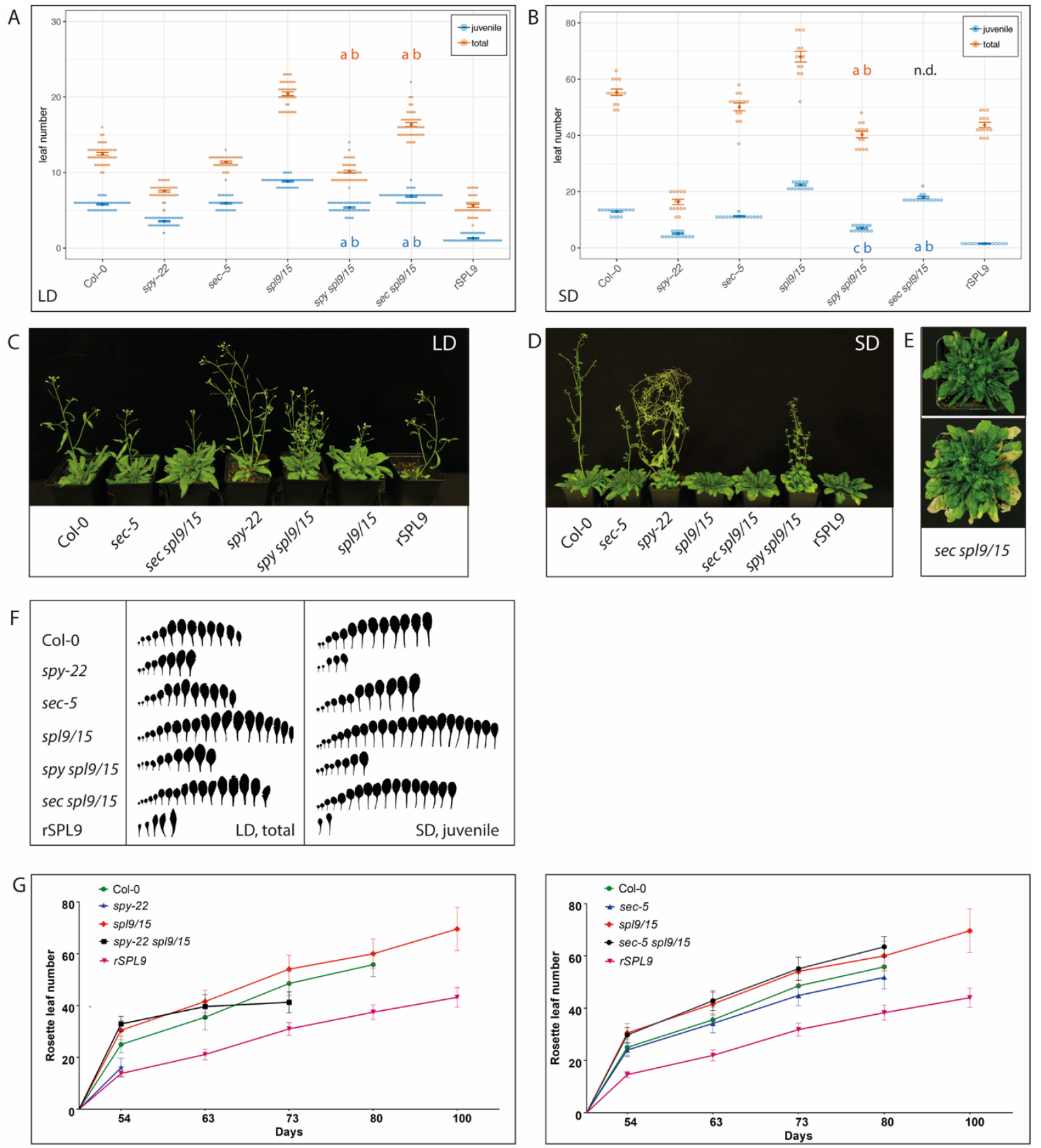

Figure 3. SPY regulates phase transitions via SPL transcription factors.

(A, B) Juvenile and total rosette leaf numbers of plant lines indicated in the graph, grown in LD (A), and SD (B) conditions. (C, D) Representative pictures of plants with leaf numbers shown in (A) and (B). (E) Magnification of representative sec sp/9/15 plants grown in SD conditions, with multiple rosette leaf branches formed before bolting of the primary inflorescence. (F) Scans of total rosette leaves of plants grown in LD conditions, and juvenile leaves of plants grown in SD conditions, plant lines as indicated in the figure. (G) Leaf growth rates of the SD-grown plants quantified in (B).

For comparison of rosette leaf numbers, one-way ANOVA with Tukey's multiple comparison was done, $\mathrm{n}$ is given in Table 3 . 

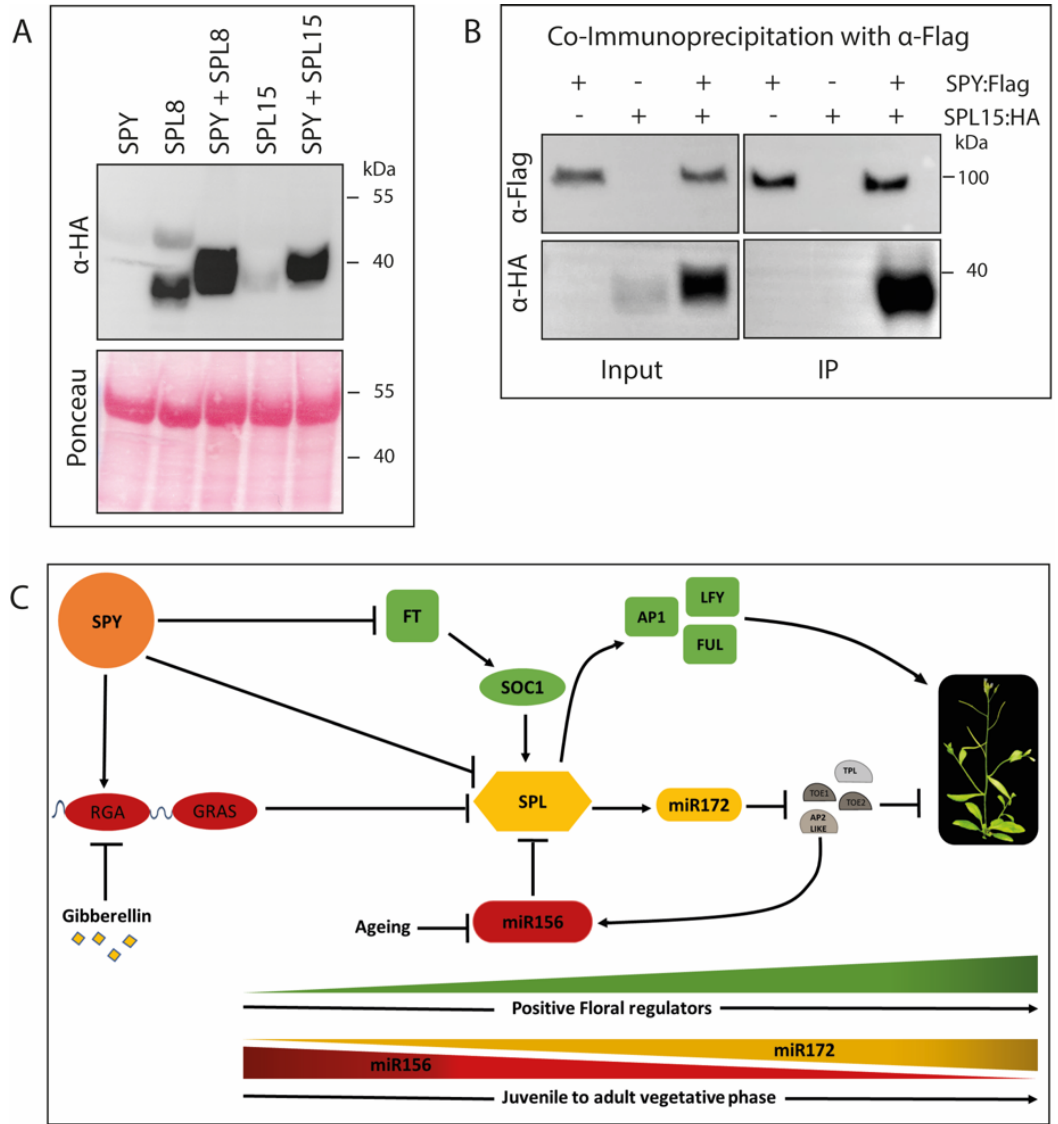

Figure 4. SPY interacts with SPLs.

(A) Western Blot of protein extracts from transient expression in Nicotiana benthamiana, 35S::SPY:Flag, 35S::SPL8:HA, 35S::SPL15:HA single infiltrations, as well as 35S::SPY:Flag, 35S::SPL8:HA and 35S::SPY:Flag, 35S::SPL15:HA co-infiltration is shown. Protein extracts were blotted and probed with anti HA-antibody to visualize SPL8 and SPL15. (B) Samples after co-infiltration of transiently expressed 35S::SPY:Flag and 35S::SPL15:HA were used for immunoprecipitation of SPY using an anti-Flag antibody, co-immunoprecipitation of SPL15 is shown with an anti-HA antibody. (C) Current working model suggesting a role for O-glycosylation at several levels in the regulation of developmental transitions in Arabidopsis thaliana.
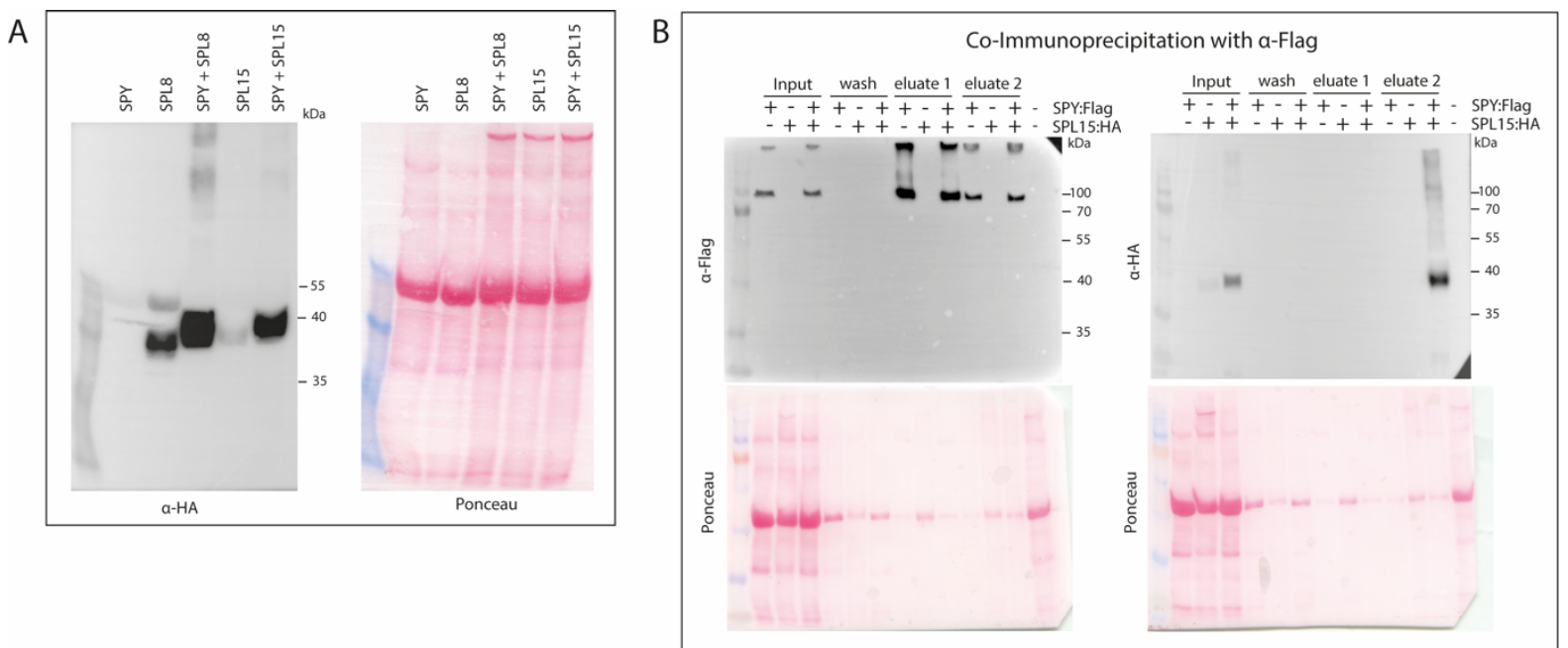

\section{Supplemental Figure 4.}

Full membranes and Ponceau staining of Western Blots shown in Figure 4. 